\title{
Nuevos retos en la formación especializada. I. Perfil y evaluación del Jefe de Estudios. II. Plan de calidad de la docencia en los centros sanitarios. III. Evaluación del residente. Propuesta de evaluación de las rotaciones
}

\author{
M. Ezquerra a , F. Pérez-Iglesias, V. Garrigues, J.M. Muñoz, J.M. Fornells, A. Gual, \\ en representación de los participantes en el VI Encuentro de Tutores y Jefes de Estudio
}

La misión de AREDA es promover e impulsar actividades destinadas a mejorar la formación de especialistas en ciencias de la salud, principalmente a través de las actuaciones realizadas por las diferentes Redes Autonómicas de Comisiones de Docencia que la componen. Entre sus objetivos se encuentra: generar opinión y difundirla; elaborar propuestas específicas de actuación y vehiculizarlas hacia las instituciones, los organismos y las personas responsables de ejecutarlas; y promover la investigación en formación sanitaria especializada (FSE).

EI VI Encuentro de Tutores y Jefes de Estudio abordó nuevos retos en la Formación Especializada, analizando específicamente el nuevo perfil de los residentes; el perfil y la evaluación del Jefe de Estudios; los Planes de Calidad de la Docencia; y la Evaluación de los Residentes.

\section{Perfil y evaluación del Jefe de Estudios}

\section{Consideraciones previas}

Modelos existentes en diferentes centros y comunidades autónomas

Variabilidad organizativa y estructural que dificulta las generalizaciones.

Funciones y responsabilidades de los Jefes de Estudios derivadas de la nueva ordenación formativa

Nueva denominación: Jefe de Estudios de formación especializada.

Según la LOPS (Anexo 1), las funciones del Jefe de Estudios tienen la consideración de funciones de gestión clínica.

La relación de funciones de la Comisión de
Docencia y del Jefe de Estudios se presentan en apartados diferentes en el Real Decreto 183/2008 (Anexo 2) y en la Orden SCO 581/2008 (Anexo 3). Cuestión a considerar: relación del Jefe de Estudios con la comisión de docencia.

\section{Variaciones en las funciones del Jefe de Estudios entre los distintos centros docentes}

La denominación de 'Jefe de Estudios' se amplía a Unidades Docentes de Especialidades Médicas (Medicina de Familia, Medicina del Trabajo, Salud Mental, Medicina Preventiva y Salud Pública, etc.) y de Enfermería (nuevas especialidades reconocidas)

El nuevo marco normativo puede condicionar las labores del Jefe de Estudios en los diferentes tipos de centros y situaciones.

\section{a Presidenta de AREDA. E-mail mezquerra@cst.cat}

Este trabajo es fruto del VI Encuentro de Tutores y Jefes de Estudios organizado por AREDA y SEDEM, celebrado en Menorca del 25 al 26 de septiembre de 2008 


\section{Recomendaciones y conclusiones de consenso}

\section{Capacidad de gestión y condiciones precisas para desempeñar las funciones del Jefe de Estudios}

Puesto que la Docencia debe considerarse un área de gestión estratégica al máximo nivel, la garantía de los recursos necesarios para desarrollar sus labores debe reflejarse en el plan estratégico docente del centro (y específicamente en el plan de calidad de la Docencia): estructura docente, recursos materiales y recursos humanos (técnicos de formación, personal administrativo, etc.) definidos:

- En los recursos necesarios para el desempeño de la labor del Jefe de Estudios es especialmente importante garantizar la adecuada dedicación de los Tutores.

- De acuerdo con las características de gestión de la Docencia, el Jefe de Estudios debe ejercer su responsabilidad docente en una situación de superioridad jerárquica respecto a las jefaturas asistenciales.

\section{Recomendaciones en cuanto a la dedicación del Jefe de Estudios para cumplir sus funciones}

- Dedicación adecuada al nivel de complejidad del centro y de las áreas de responsabilidad asignadas, lo que debe ser establecido formalmente. Debe tenderse al mayor nivel de dedicación posible. La dedicación completa es deseable en grandes centros.

- El Jefe de Estudios no debe tener responsabilidades de gestión, particularmente de tipo asistencial, en otras áreas distintas de la Docencia.

- La profesionalización de la Docencia debe ser reconocida a nivel de carrera profesional y salarial, de acuerdo con objetivos pactados en la institución.

\section{Aproximación al perfil recomendable para el adecuado ejercicio de la jefatura de estudios}

Perfil profesional (aspectos deseables y adaptados al tipo de centro):

- Especialista en Ciencias de la Salud por el sistema de residencia.
- Trayectoria profesional previa: deseable experiencia asistencial suficiente.

- Experiencia acreditada en formación de especialistas: tutor de residentes, colaborador docente...

- Competencia y liderazgo adecuados en gestión de equipos humanos.

- Conocimiento adecuado del centro docente (no excluyente).

\section{Otros aspectos}

- Designación y nombramiento: Importancia de considerar el criterio y la conformidad de la Comisión de Docencia.

- Carrera profesional: Reconocimiento de la misma durante el periodo en que se desempeñe la labor de Jefe de Estudios.

- Permanencia en el puesto: En función de evaluación del desempeño, labor y nivel de cumplimiento de objetivos, balance de gestión anual o periódico, en función de lo establecido en la LOPS.

- En cada centro deben establecerse criterios de evaluación del desempeño, en cuya definición participará la Comisión de Docencia.

\section{Consideraciones finales}

De acuerdo con la normativa vigente, el grupo de trabajo realiza las siguientes recomendaciones:

- Parece imprescindible consensuar unos acuerdos mínimos sobre la figura del Jefe de Estudios desde la perspectiva y experiencia de los jefes de estudios que han tenido esta responsabilidad hasta ahora; esto puede servir de apoyo a las distintas comunidades autónomas en la regulación que obligatoriamente deben realizar en el plazo de un año a partir de la publicación del Real Decreto.

- Importancia de la autonomía de gestión.

- Dependencia jerárquica coherente dentro de la organización (director gerente o equivalente o superior).

- Recursos humanos y estructurales para el desempeño de las funciones que se marcan en la Orden, y en función del tamaño de la Unidad (número de residentes, de especialidades, etc) y de la complejidad de la organización sanitaria donde se encuentre. 


\section{Anexos}

Anexo 1. Ley 44/2003, de 21 de noviembre, de ordenación de las profesiones sanitarias

Puntos en que se menciona la función del Jefe de Estudios:

Artículo 10. Gestión clínica en las organizaciones sanitarias

1. Las Administraciones sanitarias, los servicios de salud o los órganos de gobierno de los centros y establecimientos sanitarios, según corresponda, establecerán los medios y sistemas de acceso a las funciones de gestión clínica, a través de procedimientos en los que habrán de tener participación los propios profesionales. Tales funciones podrán ser desempeñadas en función de criterios que acrediten los conocimientos necesarios y la adecuada capacitación.

2. A los efectos de esta ley tienen la consideración de funciones de gestión clínica las relativas a la jefatura o coordinación de unidades y equipos sanitarios y asistenciales, las de tutorías y organización de formación especializada, continuada y de investigación y las de participación en comités internos o proyectos institucionales de los centros sanitarios dirigidos, entre otros, a asegurar la calidad, seguridad, eficacia, eficiencia y ética asistencial, la continuidad y coordinación entre niveles o el acogimiento, cuidados y bienestar de los pacientes.

3. El ejercicio de funciones de gestión clínica estará sometido a la evaluación del desempeño y de los resultados. Tal evaluación tendrá carácter periódico y podrá determinar, en su caso, la confirmación o remoción del interesado en dichas funciones, y tendrá efectos en la evaluación del desarrollo profesional alcanzado.

4. El desempeño de funciones de gestión clínica será objeto del oportuno reconocimiento por parte del centro, del servicio de salud y del conjunto del sistema sanitario, en la forma en que en cada comunidad autónoma se determine.

Anexo 2. Real Decreto 183/2008,

de 8 de febrero, por el que se determinan

y clasifican las especialidades en Ciencias

de la Salud y se desarrollan determinados aspectos

del sistema de formación sanitaria especializada

(BOE 45: 21 Feb 2008)

Puntos en que se menciona específicamente al Jefe de Estudios:
CAPÍTULO III (Órganos docentes de carácter colegiado: comisiones de docencia)

Artículo 10 (Composición, funciones y presidencia de las comisiones de docencia)

2. La presidencia de las comisiones de docencia la ostentará el Jefe de Estudios de formación especializada al que corresponderá la dirección de las actividades de planificación, organización, gestión y supervisión de la docencia especializada, por lo que las comunidades autónomas garantizarán su adecuada capacitación regulando el procedimiento para su designación y desempeño, en el marco de lo previsto en el artículo 10 de la Ley 44/2003 de 21 de noviembre, y con sujeción a los criterios comunes que fije la Comisión de Recursos Humanos del Sistema nacional de Salud

CAPÍTULO V (Deber general de supervisión y responsabilidad progresiva del residente)

Artículo 15 (La responsabilidad progresiva del residente)

5. Las comisiones de docencia elaborarán protocolos escritos de actuación para graduar la supervisión de las actividades que lleven a cabo los residentes en áreas asistenciales significativas, con referencia especial al área de urgencias o cualesquiera otras que se consideren de interés. Dichos protocolos se elevarán a los órganos de dirección del correspondiente centro o unidad para que el Jefe de Estudios de formación especializada consensúe con ellos su aplicación y revisión periódica.

\section{CAPÍTULO VI (Evaluación).}

Artículo 19 (Comités de evaluación)

2. Los comités de evaluación estarán integrados, al menos: a) por el Jefe de Estudios de formación especializada, que presidirá el comité y dirimirá con su voto los empates que pudieran producirse (...).

Artículo 23. Publicación de las evaluaciones anuales y sus efectos

2. Efectuadas las evaluaciones anuales, los comités de evaluación trasladarán sus resultados a la comisión de docencia, que insertará en el tablón oficial de anuncios una reseña, firmada por su presidente, para que en el plazo de 10 días puedan consultarse (...).

3. Transcurrido el plazo de diez días desde que se pu- 
blique la reseña que se cita en el apartado anterior, el presidente de la comisión de docencia convocará a los respectivos comités de evaluación, trasladándoles las evaluaciones positivas del último año y las negativas del último año no recuperables por no haberse formulado solicitud de revisión, para que con carácter inmediato procedan a llevar a cabo las evaluaciones finales.

Artículo 25. La evaluación final del periodo de residencia

3. Los comités de evaluación trasladarán las evaluaciones finales a la comisión de docencia que publicará en su tablón de anuncios una reseña, firmada por el presidente, para que en el plazo de diez días puedan consultarse en la secretaría de la comisión, en el horario que se indique, las calificaciones obtenidas en las evaluaciones finales.

4. Transcurrido dicho plazo, el presidente de la comisión de docencia remitirá con carácter inmediato al Registro Nacional de Especialistas en Formación las evaluaciones finales y las solicitudes de revisión de las mismas.

\section{Anexo 3. Orden SCO/581/2008, de 22 de febrero, por la que se publica el Acuerdo de la Comisión de Recursos Humanos del Sistema Nacional de Salud, por el que se fijan criterios generales relativos a la composición y funciones de las comisiones de docencia, a la figura del Jefe de Estudios de formación especializada y al nombramiento del tutor}

\section{Criterios comunes relativos a las funciones del Jefe de Estudios de formación especializada}

1. Asumir la presidencia de la comisión de docencia, dirimiendo con su voto los empates que se produzcan en la adopción de acuerdos.

2. Asumir la representación de la comisión de docencia formando parte, en los términos que establezcan las comunidades autónomas, de los órganos de dirección de los correspondientes centros y servicios sanitarios, con el fin de asegurar y garantizar la incardinación de la docencia en la actividad asistencial ordinaria, continuada y de urgencias de dichos centros.

3. Dirigir y coordinar las actividades de los Tutores y actuar como interlocutor con los responsables de todas las unidades docentes.

4. Actuar como interlocutor entre los responsables asistenciales y docentes con la finalidad de garantizar una adecuada coordinación entre los mismos.
5. Consensuar y suscribir con los correspondientes órganos de dirección del centro en representación de la comisión de docencia, el protocolo de supervisión de los residentes según la legislación vigente

6. Presidir, según prevé la legislación vigente, los correspondientes comités de evaluación anual, dirimiendo con su voto los empates que pudieran producirse.

7. Supervisar el plan de gestión de calidad docente del centro o unidad.

8. Promover, fomentar y definir líneas y actividades de investigación, relacionadas con las especialidades en ciencias de la salud en consonancia con los planes de salud de la comunidad autónoma y los programas I $+\mathrm{D}$, relacionados con la formación sanitaria especializada.

9. Garantizar la correcta remisión, en tiempo y forma, de las evaluaciones y demás documentación que se deba trasladar al Registro de Especialistas en Formación del Ministerio de Sanidad y Consumo.

10. Gestionar los recursos humanos y materiales asignados a la comisión de docencia, elaborando el plan anual de necesidades según la normativa aplicable en cada comunidad autónoma.

11. Ordenar la inserción en el tablón de anuncios de los avisos y resoluciones de la comisión de docencia que requieran publicación insertando la diligencia relativa a la fecha de publicación que en cada caso corresponda.

12. Aquellas otras que le asigne la correspondiente comunidad autónoma y demás normas que regulen la formación sanitaria especializada.

IV. Criterios comunes respecto a la designación, evaluación y reconocimiento de la figura del Jefe de Estudios de formación especializada

1. El jefe de estudios de formación especializada será designado en los términos que determine cada comunidad autónoma, con sujeción a lo previsto en el artículo 10. 1 de la Ley 44/2003, de 21 de noviembre.

2. Teniendo en cuenta las funciones de organización de la formación sanitaria especializada que corresponden a los jefes de estudios de formación especializada y de acuerdo con lo previsto en el artículo 10, apartados 2, 3 y 4, de la Ley 44/2003, de 21 de noviembre, dichas funciones serán consideradas de gestión clínica y como tales deben ser evaluadas y reconocidas, correspondiendo a las comunidades autónomas regular los procedimientos necesarios de reconocimiento, incentivación y evaluación periódica, de dichas funciones. 


\section{Plan de calidad de la docencia en los centros sanitarios}

El objetivo general de este apartado es presentar una propuesta de mínimos que sea aceptable para el conjunto de Centros Sanitarios y Unidades Docentes del país como ayuda para desarrollar e implantar un Plan de Gestión de la Calidad Docente.

El documento se basa en la normativa más reciente que regula los aspectos formativos de la FSE, que se presenta someramente y se estructura en varios objetivos específicos:

- Identificar qué actividades docentes requieren Planes de Gestión de Calidad y cuáles de ellas deben ser abordadas por AREDA.

- Identificar los órganos y personas que deben liderar y colaborar en la elaboración e implantación de los Planes de Gestión de Calidad de la Docencia y su relación con el organigrama de los Centros.

- Definir los elementos básicos que deben componer el Plan.

- Definir la interrelación que debe existir entre los Planes de Gestión de Calidad de la Docencia de cada Centro y el Plan Nacional de Auditorías Docentes del Ministerio de Sanidad y Consumo, así como el papel de las Comunidades Autónomas a este respecto.

- Decidir dónde y cómo difundir este documento.

\section{Desarrollo de los planes de calidad de la docencia}

\section{Introducción}

La existencia de un Plan de Calidad es necesario para conseguir avanzar hacia la excelencia en la Docencia en los Centros sanitarios, de lo contrario las acciones realizadas suelen ser incoordinadas, a menudo carecen de objetivos estratégicos y su desarrollo e impacto es difícil de evaluar.

Por otro lado, la mera existencia de un Plan no asegura su éxito. Es necesario que los objeti- vos estratégicos sean factibles y de impacto; que las iniciativas sean realizables y ayuden realmente a conseguir los objetivos; y que los indicadores sean medibles y objetivos.

Además, es imprescindible que el Plan de Calidad de la Docencia esté en línea con la estrategia del centro y que los órganos y personas que desarrollan el plan estén en sintonía con el organigrama directivo del centro. Asimismo, también es imprescindible que el equipo directivo del Centro preste su apoyo decidido a los responsables de Docencia en el desarrollo de sus Planes Estratégicos y de Calidad.

Un punto importante a tener en cuenta es la variabilidad existente entre los diferentes Centros sanitarios, tanto en tamaño, estructura y función como en el deseo de sus responsables en organizar las actividades docentes de una determinada forma. Ello implica que los Planes de Gestión de la Calidad de la Docencia en los diferentes Centros deban ser necesariamente diferentes. Sin embargo, sería muy deseable conseguir un cierto grado de homogeneidad, lo que iría en beneficio de todos, facilitando el intercambio de información y experiencias.

Otro punto de importancia es que los Planes de Calidad deberían estar en línea con el Plan Nacional de Auditorías Docentes del MSC o con cualquier otro Plan de Auditorías externas dependiente del gobierno central o de las Comunidades Autónomas. Ello otorgaría a los Planes de Calidad una mayor potencia para conseguir su objetivo final.

\section{Normativa aplicable}

El Real Decreto 183/2008, de 8 de febrero, por el que se determinan y clasifican las especialidades en Ciencias de la Salud y se desarrollan determinados aspectos del sistema de formación sanitaria especializada, en su Artículo 29.3 indica que las comisiones de docencia de centro o unidad elaborarán un plan de gestión de calidad docente respecto a la/s unidad/es de las distintas especia- 
Tabla. Objetivos (obj) e indicadores (ind) que se proponen como mínimos para una Plan de Gestión de la Calidad de la Docencia.

Obj 1. Satisfacer las necesidades y expectativas docentes de los residentes.

- Ind 1.1 Índice de satisfacción global de los residentes.

- Ind 1.2 Índice de satisfacción de los residentes con la formación recibida en las rotaciones por los diferentes servicios.

Obj 2. Mejorar el nivel de atracción del Centro/Unidad Docente.

- Ind 2.1 Número de residentes en el primer cuartil, o similar.

Obj 3. Elaborar guías/itinerarios formativos para cada especialidad acreditada, que garanticen el cumplimiento de los objetivos del programa oficial de la especialidad.

- Ind 3.1 Porcentaje de especialidades con guía formativa actualizada.

Obj 4. Elaborar planes individuales de formación para cada residente, adecuados a la guía/itinerario formativa de su especialidad y a sus características personales.

- Ind 4.1 Porcentaje de residentes con plan individual de formación.

Obj 5. Fomentar la participación de los residentes en cursos, congresos, seminarios o reuniones científicas, relacionados con su especialidad.

- Ind 5.1 Número de comunicaciones presentadas por residente.
Obj 6. Asegurar la formación en competencias trasversales.

- Ind 6.1 Porcentaje de residentes que asisten al Programa de Formación Común (PFC).

- Ind 6.2 Índice de satisfacción de los residentes con el PFC.

- Ind 6.3 Porcentaje de residentes cuyo programa incluye

la rotación por Atención Primaria que lo realizan.

Obj 7. Promover la participación de los residentes en proyectos de investigación.

- Ind 7.1 Porcentaje de residentes que han participado en al menos una publicación.

- Ind 7.2 Porcentaje de residentes que han participado en al menos un proyecto de investigación.

Obj8.ProfesionalizarlafuncióndelosTutoresderesidentes.

- Ind 8.1 Porcentaje de Tutores formados en metodología docente.

- Ind 8.2 Porcentaje de Tutores acreditados.

Obj 9. Realizar evaluación formativa a los residentes.

- Ind 9.1 Porcentaje de residentes con evaluación formativa trimestral realizada.

- Ind 9.2 Porcentaje de residentes con libro del residente completado, visado y aprobado.

Obj 10. Difundir el Plan de Gestión de la Calidad de la Docencia y sus resultados a todos los profesionales del Centro/Unidad Docente.

- Ind 10.1 Porcentaje de residentes que afirman conocer el Plan. lidades que se formen en su ámbito. Dicho plan se aprobará y supervisará por la Comisión de Docencia con sujeción a los criterios que establezcan las comunidades autónomas a través de los órganos competentes en materia de formación sanitaria especializada y la Agencia de Calidad del Sistema Nacional de Salud.

La Orden SCO/581/2008, de 22 de febrero, por la que se publica el Acuerdo de la Comisión de Recursos Humanos del Sistema Nacional de Salud, por el que se fijan criterios generales relativos a la composición y funciones de las Comisiones de Docencia, a la figura del Jefe de Estudios de formación especializada y al nombramiento del tutor, indica que es función de las Comisiones de Docencia aprobar el plan de gestión de calidad docente del centro o unidad docente, supervisando su cumplimiento, a cuyos efectos les será facilitada cuanta información sea necesaria por los responsables de las unidades asistenciales y por los correspondientes órganos de dirección y gestión.

Asimismo, esta Orden indica que es función del Jefe de Estudios supervisar el plan de gestión de calidad docente del Centro o Unidad.

\section{Actividades docentes que requieren Planes de Gestión de Calidad}

Idealmente, todas las actividades docentes que se realicen en un determinado Centro o Unidad deberían estar incluidas en los Planes de Gestión 
de Calidad que, por tanto, deberían considerar la formación de grado y postgrado de las distintas profesiones relacionadas con la salud; la formación especializada de facultativos y enfermería; y la formación continuada de los profesionales del centro.

Por ello, los clientes internos para los Planes de Calidad de la Docencia serían los estudiantes, los residentes y los profesionales. Además, debería considerarse también la posibilidad de incluir como cliente externo a la población, cuya asistencia sanitaria depende de cada centro.

Dada la naturaleza y objetivos de AREDA, las recomendaciones de este documento se ciñen exclusivamente a la formación especializada. En cada Centro o Unidad deberá decidirse, según sus características y posibilidades si el Plan se extiende al resto de actividades docentes.

Órganos y personas que deben liderar y colaborar en la elaboración y desarrollo de los Planes de Gestión de Calidad de la Docencia y su relación con el organigrama de los Centros

El equipo directivo de los centros debe estar necesariamente implicado en el proyecto, liderando, estimulando y facilitando su puesta en marcha, dado que el desarrollo del Plan de Gestión de la Calidad de Docencia debe realizarse en línea con el Plan de Gestión de Calidad Total propio de cada Centro. Debe incluirse objetivos docentes en los Contratos/Pactos de Gestión/ Programa acordados con las Unidades Clínicas

Además, se considera imprescindible la participación activa de expertos en Gestión de Calidad. Asimismo se considera que debe ponerse a disposición de los responsables de docencia cualquier recurso que pudiera resultar necesario.

El Plan de Gestión de Calidad de la FSE debe elaborarse en el seno de las Comisiones de Docencia. El Presidente de la citada Comisión debe liderar el proyecto, en el que deben participar activamente todos sus miembros, especialmente los representantes de los Tutores y de los Residentes y, a través de ellos, las personas a las que representan.

Sin embargo, en aquellos centros en los que se decida elaborar Planes de Calidad también para la formación de grado-postgrado y para la formación continuada, debería debatirse quiénes deben ser los responsables de la actividad, en función de las características de cada Centro y de su experiencia previa. En algunos casos, la propia Comisión de Docencia podría responsabilizarse de esta tarea; pero en otros podría ser más apropiado crear un órgano diferente que, en colaboración con la Comisión de Docencia, abordará los aspectos no relacionados con la FSE y coordinara todas las actividades.

\section{Definir los elementos que deben formar el Plan}

Se considera que cualquier modelo de Gestión de Calidad (EFQM, ISO 9000, Joint Comission, o cualquier otro) puede resultar adecuado. La elección depende de la experiencia acumulada en cada Centro o Unidad Docente.

Es imprescindible que todos los componentes del Plan sean documentados ampliamente. Específicamente, es necesario que los objetivos estratégicos y sus indicadores sean definidos con claridad.

También podría ser útil que se documentara ampliamente el proceso de FSE, desde la acogida hasta la despedida del residente, incluyendo todas las actividades formativas trasversales, específicas de cada especialidad y externas.

Los elementos esenciales del Plan de Gestión de Calidad deberían ser:

\section{La Misión, Visión y Valores docentes del Cen-} tro o Unidad Docente

La Misión docente es el motivo principl para el que el Centro/Unidad realiza actividades formativas.

La Visión docente consiste en establecer el punto donde se quiere llegar en relación con las actividades formativas.

Los Valores son los recursos de excelencia con los que se cuenta.

\section{Los objetivos estratégicos docentes}

Cada Centro o Unidad Docente debe establecer los objetivos docentes más apropiados para sus características.

Cada objetivo seleccionado debe ser fácilmente medible, mediante al menos un indicador. Se sugiere la inclusión en el Plan de unos objetivos mínimos (Tabla).

\section{Los indicadores y sus metas}

Los indicadores elegidos deben ser objetivos y fáciles de obtener. Debe establecerse una meta a alcanzar para cada uno, con una periodicidad al menos anual. 
Se propone una serie de indicadores (Tabla), que se corresponden con los objetivos docentes propuestos en el punto anterior.

\section{El sistema de evaluación}

Comprende la evaluación interna y la externa.

La evaluación interna debe ser realizada por la Comisión de Docencia, verificando el cumplimiento de los objetivos a través del análisis de los indicadores, con una frecuencia al menos anual. El resultado de la evaluación debe ser validado por la Dirección del Centro o Unidad Docente y hecho público.

La evaluación externa se basará en el Plan de Calidad del Sistema Nacional de Salud y/o Comunidades Autónomas.

\section{Las iniciativas y acciones de mejoras}

Estas iniciativas y acciones de mejora se derivan del resultado de la evaluación interna o externa. Son necesarias para corregir las deficiencias observadas.

Definir la interrelación que debe existir entre los Planes de Calidad de cada Centro y el Plan Nacional de Auditorías Docentes del Ministerio de Sanidad y Consumo, así como el papel de las Comunidades Autónomas a este respecto

La elaboración, desarrollo y mantenimiento de un Plan de Gestión de la Calidad es una tarea ardua, que requiere de la dedicación continuada de un número no despreciable de personas. Por ello, es importante que de este esfuerzo se derive la mayor eficiencia posible.

Específicamente, debería existir alguna interrelación entre los centros que elaboren Planes de Calidad de la Docencia y las administraciones, autonómicas o estatal, a fin de que los planes se elaboren teniendo en cuenta los criterios de calidad utilizados por las administraciones $y$, a su vez, que estos criterios de calidad tengan en cuenta también a los planes desarrollados en los centros.

Decidir dónde y cómo difundir las conclusiones del documento

Este es un aspecto muy importante a tener en cuenta ya que en buena medida el éxito o fracaso del documento depende de su difusión y del impacto de sus conclusiones.

Se propone que además de la difusión entre los asistentes al Encuentro y entre los miembros de AREDA, las conclusiones sean remitidas a los responsables de docencia del Ministerio de Sanidad y Consumo, de las Comunidades Autónomas y de los Centros/Unidades Docentes. Además, debería realizarse una difusión a través de los medios de comunicación profesionales.

\section{Evaluación del residente. Propuesta de evaluación de las rotaciones}

En relación con la evaluación del residente la publicación del BOE de febrero de 2008 aportará un nuevo marco legal, que en estos momentos tiene pendientes de desarrollar algunos puntos. El objetivo general de este grupo de trabajo ha sido comentar el nuevo marco legal y valorar una propuesta de modificación de la evaluación de las rotaciones. Los participantes en el Encuentro se enfrentaron a los siguientes objetivos específicos:
- Identificar las modificaciones que en relación a la evaluación comporta el nuevo marco legal.

- Definir los elementos clave para cualquier procedimiento de evaluación.

- Establecer los objetivos de la evaluación de las diferentes rotaciones y contextualizarlos en el proceso de evaluación del residente.

- Valorar las listas de comprobación como instrumento de evaluación de las rotaciones. 
- Decidir dónde y cómo difundir las conclusiones de AREDA al respecto.

\section{Marco legal de la evaluación del residente}

El Real Decreto 183/2008 establece una nueva normativa en relación a la evaluación del residente, a continuación se exponen los puntos que se deben destacar:

- Establece dos tipos de evaluación: la formativa y la sumativa.

- Evaluación formativa:

- Instrumentos de evaluación:

- Entrevistas periódicas tutor-residente en número no inferior a 4 al año. Se deben registrar en el libro del residente y en los informes de evaluación formativa.

- Otros instrumentos que permitan una valoración objetiva del progreso competencial.

- Libro de residente como soporte al proceso de evaluación formativa.

- Informes de evaluación formativa: Informe que realizará el tutor, formato normalizado por el Ministerio de Sanidad y Consumo.

- Libro del residente: Carácter obligatorio, registro de las actividades que evidencian el proceso de aprendizaje, se tendrán en cuenta en la evaluación del proceso formativo. Propiedad del residente. Cada Comisión Nacional diseñará la estructura básica del libro del residente.

- Comité de evaluación.

- Evaluación sumativa anual:

- Calificación anual del residente en positiva o negativa, las evaluaciones negativas podrán ser recuperables o no recuperables:

- El informe anual del tutor es el instrumento básico para la valoración del progreso anual del residente en el proceso de adquisición de competencias profesionales. Debe contener:

- Informes de evaluación formativa (informes de las rotaciones, informes de otras pruebas objetivas, participación en cursos y congresos).

- Informes de las rotaciones externas.

- Informes que se soliciten de los jefes de las distintas unidades asistenciales.

- En un plazo de 10 días posteriores a la publicación de las calificaciones se podrá solicitar la revisión de las evaluaciones negativas no recuperables y de las recuperables al finalizar el período de recuperación si han sido negativas.

\section{- Evaluación sumativa final:}

- Tiene como objeto verificar que el nivel de competencias adquirido por el especialista en formación durante todo el período de la residencia. Se valorará el expediente completo.

- La calificación podrá ser positiva, positiva destacado y negativa.

- Los residentes que obtengan la calificación de positiva o positiva destacado podrán solicitar su revisión ante la Comisión Nacional de su especialidad, para optar a la calificación de destacado con mención o destacado con mención especial mediante la realización de una prueba.

- En un plazo de 10 días posteriores a la publicación de las calificaciones se podrá solicitar la revisión de las evaluaciones finales positivas y negativas ante la comisión nacional de la especialidad correspondiente.

- En caso de evaluación negativa la comisión nacional realizará una prueba de evaluación al residente que lo solicite. Si esta fuera negativa podría solicitar la realización de una prueba extraordinaria, entre los 6 meses como mínimo y un año, el resultado de esta sería el definitivo.

- En caso de evaluación positiva la comisión nacional realizará una prueba de evaluación al residente que lo solicite para optar a la calificación de destacado con mención de la CNE y destacado con mención especial de la CNE.

- El Ministerio de Sanidad y Consumo, previo informe de la Comisión de Recursos 
Humanos del Sistema Nacional de Salud teniendo en cuenta el Real Decreto 183/2008 y los criterios de evaluación que determinen las comisiones nacionales de la especialidad, aprobará las directrices básicas que deben contener los documentos acreditativos de las evaluaciones.

- El plazo de adaptación de la normativa es de un año desde la publicación del Real Decreto $183 / 2008$. Los residentes que hayan obtenido plaza en formación en convocatorias anteriores a la fecha en que se aprueben dichas directrices seguirán siendo evaluados según lo previsto en la Orden del 22 de junio de 1995. (Si se cumplen los plazos afectará a los residentes a partir de la promoción 2009-2010.

\section{Elementos clave para cualquier procedimiento de evaluación}

\section{Condiciones necesarias previas}

- Un Programa Oficial (POE) que tenga definidas las competencias a adquirir y los estándares aceptables de actuación en la práctica, esto permitirá definir que es lo que se quiere evaluar.

- Control sobre la aplicación del programa en cada Unidad Docente: es necesario un control sobre lo que se esta enseñando desde un punto de vista práctico.

- Tutores acreditados y reacreditados con formación metodológica y una dedicación suficiente a la labor educativa.

- Centros docentes que supervisen la labor de los Tutores y un mecanismo de control sobre los centros docentes.

- La aceptabilidad por parte de los protagonistas implicados (discentes y docentes incluyendo la estructura docente).

- La practicabilidad o viabilidad, diseñar un sistema adecuado a los recursos disponibles.

\section{Valoración formativa}

\section{Objetivos}

El objetivo de esta evaluación es la valoración del progreso en el proceso de aprendizaje del residente, medir la competencia adquirida de forma progresiva en relación a los objetivos planteados e identificar las áreas susceptibles de mejora y aportar sugerencias específicas para alcanzarlos.

\section{Características de la evaluación formativa}

- Continuada, a lo largo de todo el periodo de formación.

- Debe tener como eje central la relación entre el tutor y el residente en formación.

- Se debe dar un feedback constructivo sobre la actuación del residente de forma regular y planificar conjuntamente la estrategia docente, con el objetivo de garantizar las instrucciones y los cambios necesarios para mejorar el desarrollo de las competencias.

- Los métodos de evaluación deberían estimular un aprendizaje integrado y evaluar requerimientos prácticos predefinidos, así como también conocimientos, aptitudes y actitudes.

- Además de contar con elementos subjetivos por parte de los Tutores y colaboradores docentes se deben utilizar métodos objetivos de evaluación.

- Se deben establecer mecanismos que permitan la modificación de la aplicación práctica del programa de formación en función de los resultados de la evaluación formativa.

\section{Evaluación sumativa final}

\section{Objetivos}

- Constatar el grado de aprovechamiento alcanzado en relación al Programa de Formación.

- Clasificar a los residentes y establecer puntos de corte que definan el grado de aprovechamiento mínimo necesario para poder superar la prueba o pruebas que la componen.

- Permite certificar la aptitud o la falta de aptitud para desarrollar una actividad profesional autónoma.

\section{Necesidad y justificación}

- Para garantizar socialmente que los profesionales formados tienen un nivel de competencia suficiente y proteger por tanto a los 
pacientes de los profesionales cuyo nivel de competencia no es el adecuado.

- Para conocer el nivel de competencia de los profesionales en la fase inicial del ejercicio de su profesión.

- Para identificar los profesionales que todavía no están preparados y precisan complementar su formación.

- Como control de calidad del las estructuras docentes, de los programas de formación.

\section{Características}

- Debe haber una evaluación al final de los Programas de Formación de especialistas.

- El procedimiento debe tener unas características comunes para todos los profesionales en formación pero no ser exactamente igual, esto esta ampliamente justificado por las diferencias existentes en el contenido a evaluar.

- La evaluación sumativa debe ser común a todos los profesionales en formación de la misma especialidad.

- La clasificación de los profesionales que implicaría la evaluación sería entre aptos y no aptos. El establecer distintos niveles de calificación entre los residentes aptos sería un objetivo secundario teniendo en cuenta la diferente trascendencia social y profesional que representa, además comporta una mayor complejidad metodológica.

- El procedimiento debería contar con un mecanismo de revisión por parte del discente.

- El procedimiento debería prever un sistema de recuperación de los no aptos (establecer período de tiempo necesario para repetir la evaluación, requisitos, mecanismo para ampliar el periodo de formación etc.).

\section{Requisitos técnicos}

- Validez (grado en que el instrumento mide realmente lo que pretende medir).

- Fiabilidad (confianza en la precisión de los resultados).

- Reproductibilidad (la constancia en la obtención de los resultados).

- Practicabilidad (debe tener en cuenta los recursos disponibles, el tiempo, los costes, el número de discentes que se deben evaluar.)

- Relevancia de lo que se evalúa respecto a los que se debería evaluar.

\section{Evaluación de las rotaciones: situación actual}

Podríamos definir las rotaciones como una situación que permite el aprendizaje de campo (learning in context) o la formación en servicio. Consiste en colocar al residente en una situación real en la que su autoaprendizaje pueda tener lugar. Este tipo de aprendizaje esta especialmente indicado para la adquisición y aprehensión de la complejidad de las funciones y de la toma de decisiones como profesional. Tiene un alto potencial educativo, se dice que el conocimiento se recuerda mejor en el contexto que se ha aprendido. Cuenta con la observación directa pasiva de lo que los otros profesionales hacen, con la intervención directa del residente que en algunos casos tendrá una supervisión directa y en otros una supervisión diferida. En los diferentes programas formativos el aprendizaje de campo es una parte importante de la formación del residente, se determinan en que unidades asistenciales debe permanecer y el periodo de tiempo recomendado así como, los objetivos docentes y competencias a adquirir. Posteriormente cada unidad docente debe realizar la aplicación práctica del Programa. Los residentes durante este periodo de rotación tienen asignado un responsable asistencial que organiza su trabajo, los supervisa y posteriormente los evalúa.

Actualmente la evaluación de estas rotaciones se realiza siguiendo la recomendación de la Orden del 22 de junio de 1995 y las disposiciones posteriormente indicadas por el Ministerio de Sanidad. El registro que se utiliza se denomina ficha 1, es común para todas las especialidades y centros. En muchos centros y otros foros profesionales se han analizado las ventajas e inconvenientes de este sistema de evaluación e incluso de este registro. La ventaja principal del sistema es que se ha consolidado el hábito de evaluar al residente durante las diferentes rotaciones, que el tutor recibe a lo largo del año las diferentes valoraciones del residente, de forma que le permiten realizar mejor su labor de tutorización y en la mayoría de ocasiones el conjunto de valoraciones de diferentes profesionales sobre el residente permite al tutor y al comité de evaluación transformar 'la subjetividad' de que adolece este sistema. Los problemas e inconvenientes principales del sistema los analizaba la comisión de docencia del Hospital Ramon y Cajal por ejemplo: 
- Escasa aceptación de la ficha de evaluación por parte de los colaboradores docentes e incluso de muchos de los Tutores.

- Falta de interés por el propio proceso de evaluación de los residentes por la ausencia de impacto curricular de éstas. Los docentes, muchos Tutores y los propios residentes esperan herramientas de evaluación 'cuantitativas' y con 'impacto curricular'

- Hay un importante desconocimiento respecto de los procesos de evaluación de los residentes y su normativa.

- Ausencia de criterios para evaluar y de definiciones sobre lo evaluado $(i Q$ Qué es, por ejemplo 'motivación' y cómo lo evalúo?)

- Muchos docentes (aunque menos en los últimos dos años) optan por calificaciones máximas (2,9-3 sobre 3,) para 'no perjudicar' al residente respecto de 'competidores' o de residentes de otros centros (i?)

- Retraso en la recepción de fichas de evaluación respecto del momento de la realización de ésta. En alguna ocasión la recepción con retraso de evaluaciones negativas o en el límite del 'apto' no permitían gestionar adecuadamente el periodo de recuperación o las acciones de tutorización.

\section{Evaluación de las rotaciones: propuesta de futuro}

\section{Requisitos previos}

Cuando nos planteamos el tema de la evaluación de las rotaciones de los residentes tenemos que tener en cuenta una serie de elementos que son claves para lleva a buen puerto dicha evaluación.

Estos elementos los podemos agrupar en tresapartados: la planificación docente, la comunicación entre las partes y la supervisión/evaluación propiamente dicha.

\section{Planificación docente}

En lo que atañe al tutor, la primera tarea que le corresponde es la adaptación/adecuación del programa nacional de la especialidad a las características de su entorno profesional y de los residentes a su cargo.
En la situación actual nos encontramos con una gran variabilidad de los programas nacionales por lo que hace a su amplitud, profundidad y concreción. En cualquier caso, no obstante, corresponde al tutor elaborar la propuesta de programa formativo adaptado a su contexto y que deberá ser aprobado por la correspondiente comisión de docencia.

En ciertos casos, el tutor tendrá que profundizar y concretar los objetivos de aprendizaje; en otros, ante programas muy prolijos, tendrá que poner el énfasis en la necesaria priorización de las competencias a adquirir por parte del residente. No hay duda que en ciertas especialidades, los periodos de rotación pueden suponer un $25 \%$ o más del total del periodo formativo, y en consecuencia una adecuada formulación de los objetivos de aprendizaje para estos periodos, por lo general reducidos en el tiempo, es clave.

Los Tutores deberán pues formular unos objetivos generales y sobretodo específicos, que sean relevantes, concretos, claros y evaluables, que se enmarquen dentro del programa nacional y que se adapten a la duración del periodo de rotación.

En evaluación siempre decimos que si no sabemos a donde queremos llegar difícilmente podremos saber si hemos llegado. En este sentido es fácil entender que los objetivos de aprendizaje son los cimientos de cualquier sistema de evaluación. Sin objetivos no hay evaluación.

Igualmente en esta fase de planificación docente, el tutor tendrá que identificar que instrumentos de evaluación va a utilizar teniendo en cuenta la congruencia con los objetivos previamente identificados.

\section{Comunicación}

Otro aspecto básico en la evaluación de periodos de rotación es la comunicación del tutor con las diferentes partes implicadas

En primer lugar con el residente, el tutor tiene que asegurarse que el Residente conoce y entiende sus objetivos de aprendizaje en cada periodo formativo. Esta es una premisa para que se desarrolle realmente un proceso de autoaprendizaje y autoevaluación por parte del residente.

No menos importante es la comunicación del tutor con otros Tutores o facultativos responsables de las rotaciones de sus residentes en otros servicios o unidades. Se trata que los objetivos fijados por el tutor sean compartidos y asumidos por los res- 
ponsables directos de las rotaciones. El tutor debe asegurarse que aquellos objetivos esenciales de una rotación pueden ser cumplidos y para ello debe existir un dialogo, una comunicación bidireccional, NO una simple notificación de los objetivos.

\section{Supervisión/evaluación}

Es conveniente que la supervisión/ evaluación de los residentes se haga a través de formatos previamente diseñados y no se dejen a la improvisación.

A nivel de diseño se tendrá que identificar de qué forma vamos a valorar la consecución de los objetivos de aprendizaje por parte del responsable de la rotación y eventualmente del tutor. Los métodos a utilizar pues serán variables de una especialidad a otra y dependiendo siempre de lo que se quiera evaluar. Es difícil dar recetas, no obstante, algunos métodos podrían ser:

\section{Objetivos}

Evaluar las competencias que debe adquirir el residente durante la rotación por la unidad asistencial.

Detectar elementos de mejora que deberán permitir un feedback constructivo posterior por parte del tutor.

Los informes de evaluación de las rotaciones formaran parte de la evaluación formativa.

\section{¿Quién lo debe evaluar?}

Los facultativos responsables a los que esta asignado el residente durante ese periodo de rotación, habitualmente es una persona pero en rotaciones más largas pueden ser 2 o 3 personas, en ese caso si se trata de un checklist lo mejor sería rellenar uno cada responsable y elaborar un informe conjunto posteriormente. Este informe se debe remitir al tutor del residente.

En el caso de rotaciones largas es recomendable realizar una evaluación en la mitad del periodo de la rotación.

\section{Instrumento propuesto: checklist o lista de comprobación}

Consiste en la comprobación de actividades, conductas o pasos que son componentes de una competencia más compleja. La forma habitual es mediante un till o si/no que nos indica si dicha conducta o acción ha tenido lugar.

El checklist puede ser útil para evaluar aquellos componentes competenciales que se pueden partir en acciones o conductas concretas, especificas.

Hay evidencias documentadas de su utilidad para la evaluación de procedimientos clínicos, anamnesis, examen físico, o para las habilidades comunicativas. Los checklist son más útiles para proporcionar feedback dado que pueden diseñarse para evaluar acciones concretas en el cumplimiento de una tarea.

Desde el punto de vista psicométrico, la fiabilidad puede estar entre 0,7 y 0,8 (1 es el valor máximo) si los observadores están entrenados en el uso del checklist.

Para asegurar la validez de contenido y el de los criterios de puntuación es necesario desarrollar un consenso entre varios expertos. Checklist necesita evaluadores entrenados para observar la práctica y, además, tiempo para completar la lista.

Una alternativa al checklist es la global rating en los que se juzgan categorías generales y no actuaciones concretas, por ejemplo: competencia clínica, habilidades comunicativas, trabajo en equipo etc, en su conjunto. La valoración se hace retrospectivamente sobre la base de distintas observaciones para un periodo de tiempo.

Las escalas que se usan en el global rating son cualitativas que frecuentemente incluyen valores numéricos. Así, muy bien $=1$; bien $=2$; suficiente $=3$; deficiente $=4$ o también: excelente $=1$; suficiente $=2$ insatisfactorio $=3$.

La valoración con global rating se utiliza con frecuencia al final de periodos de rotación y resumiendo la evaluación de varias semanas. Los comentarios pueden complementar los valores numéricos para obtener un juicio de la actuación profesional sobre la base de más de un evaluador.

La fiabilidad vendrá condicionada por el entrenamiento de los distintos observadores.

Para la evaluación de un periodo de rotación nos podemos plantear un modelo que combine el uso de checklist con la global rating. Esto supone combinar el análisis de actuaciones concretas que supone el checklist con la valoración global de la competencia alcanzada propia de la global rating. 


\section{Formulario propuesto}

Se propone un formulario que incluye las diferentes áreas competenciales posibles, en función de los objetivos docentes se elaborarán las actividades, conductas o pasos que permitirán 'comprobar' si la competencia se ha adquirido o no.

\section{Competencias específicas}

- Competencia clínica.

- Habilidades y procedimientos.

Los objetivos de aprendizaje los adaptará el tutor según el programa de la especialidad y las características del centro.

\section{Competencias transversales}

- Comunicación con los pacientes.

- Trabajo en equipo.

- Mantenimiento de la buena práctica.

- Actitud o comportamiento profesional.

- Capacidad como docente y discente.

Los objetivos de aprendizaje de estas competencias pueden ser comunes para todos los programas de formación.

Se deben elaborar critérios de suficiencia para cada objetivo de aprendizaje.

La escala a utilizar sería cualitativa con 4 categorías: destacado/suficiente/insuficiente y no valorable.

El formulario incorporará un espacio para observaciones y comentarios.

\section{Formulario ejemplo}

\section{Competencias específicas}

Buena competencia clínica:

- Sabe hacer la anamnesis y exploración física.

- Realiza un buen planteamiento diagnóstico.

- Realiza un plan terapéutico, teniendo en cuenta la seguridad de la prescripción.

- Demuestra un apropiado manejo en la gestión del tiempo y en la toma de decisiones.

- Prioriza la seguridad de los pacientes en su práctica clínica.

- Demuestra los conocimientos, habilidades, actitudes y comportamientos para reducir el riesgo de infecciones.

- Demuestra los conocimientos, habilidades, actitudes y comportamientos para realizar las actividades de educación sanitaria pertinentes.

- Demuestra los conocimientos y habilidades para abordar los problemas éticos y legales que ocurren durante la atención prestada a los pacientes con problemas médicos generales.

- Reconocimiento y manejo clínico de las situaciones de enfermedad aguda.

Procedimientos y habilidades:

- Sabe hacer.

\section{Competencias transversales}

Relación/comunicación con los pacientes:

Demuestra los conocimientos, actitudes, habilidades y comportamientos para hacer posible una comunicación efectiva con los pacientes, sus familiares y los colegas en las diferentes situaciones: en la consulta, dando malas noticias y en las quejas y reclamaciones.

- Mantiene informado regularmente al paciente y allegados, expresándose de forma sencilla, comprensible y respetuosa.

- Escucha activamente, muestra empatía y compasión (consuelo) cuando es necesario.

- Respeta los deseos de confidencialidad del paciente y respeta su autonomía.

- Conoce y aplica el marco normativo que ampara al paciente (consentimiento informado, protección de datos, etc.).

- Muestra educación, respeto y tacto ante la diversidad cultural, social, étnica de opción sexual, etc.

- Es capaz de manejarse en situaciones conflictivas con pacientes o familiares.

\section{Trabajo con el equipo}

Demuestra las habilidades precisas para el trabajo en equipo:

- Solicita opinión y escucha con atención y respeto las opiniones y decisiones de otros profesionales.

- Expresa la discrepancia con consideración y educación. 
- Contribuye a fomentar un ambiente de trabajo agradable y saludable (dando las gracias, haciendo uso del buen humor, reconociendo la colaboración de los demás, compartiendo los méritos...).

- Asume con naturalidad y respeto las tareas que le corresponden o se le asignan.

- Muestra disponibilidad ante las necesidades del equipo, animando a la colaboración y cooperación.

\section{Mantenimiento de una buena práctica médica}

Demuestra los conocimientos, actitudes, comportamientos, habilidades y competencias necesarias para iniciar el autoaprendizaje a lo largo de su vida profesional.
- Utiliza la evidencia científica y guías clínicas.

- Demuestra los conocimientos, habilidades, actitudes y comportamientos para utilizar el audit.

\section{Comportamiento profesional}

Desarrolla los conocimientos, habilidades, actitudes y comportamientos para tener una correcta actuación profesional en la relación médico-paciente y en las situaciones de estrés.

\section{Enseñando y aprendiendo}

Demuestra los conocimientos, habilidades, actitudes y comportamientos del papel como docente. 